\title{
SPATIAL-FREQUENCY DISCRIMINATION OF DRIFTING GRATINGS
}

\author{
Mark W. GreenleE, ${ }^{1}$ Jürgen Gerling ${ }^{2}$ and Stephan Waltenspiel ${ }^{2}$ \\ Neurologische Universitätsklinik, Abt. Neurophysiologie, Hansastr. 9, 7800 Freiburg I. Br. and \\ ${ }^{2}$ Universitäts-Augenklinik, Abt. Neuroophthalmologie, Killianstr. 5, 7800 Freiburg I. Br., F.R.G.
}

(Received 17 October 1989; in revised form 8 January 1990)

\begin{abstract}
Spatial-frequency discrimination thresholds were measured for briefly $(300 \mathrm{msec})$ presented sinewave gratings having a contrast one logarithmic unit above detection threshold. The gratings were drifted at rates varying from 1.1 to $40 \mathrm{~Hz}$. In a two-interval forced-choice paradigm thresholds were determined for vertically and obliquely oriented gratings. Three reference spatial frequencies $(1,4$, $12 \mathrm{c} / \mathrm{deg}$ ) were tested. For the $1 \mathrm{c} / \mathrm{deg}$ reference spatial frequency, spatial-frequency discrimination thresholds were constant over the wide range of drift rates used. For 4 and $12 \mathrm{c} / \mathrm{deg}$ reference gratings, discrimination thresholds were constant for drift frequencies up to $14 \mathrm{~Hz}$. For drift frequencies beyond $14 \mathrm{~Hz}$, spatial-frequency discrimination thresholds increased abruptly, rising from approx. $6 \% \mathrm{at} 14 \mathrm{~Hz}$ to $25 \%$ at $40 \mathrm{~Hz}$ drift rate. Measurements with obliquely oriented gratings yielded comparable results. The increase in the spatial-frequency discrimination threshold for medium-high spatial frequencies and high temporal frequencies might reflect an increase in the spatial frequency bandwidth of the mechanisms sensitive to these stimulus frequencies.
\end{abstract}

Spatial-frequency discrimination Stimulus velocity Contrast Channels

\section{INTRODUCTION}

The human observer is capable of discriminating a difference of as little as $2-4 \%$ in the spatial frequency of two suprathreshold gratings (Campbell, Nachmias \& Jukes, 1970; Hirsch \& Hylton, 1982; Mayer \& Kim, 1986; Thomas, 1983). For a grating of high spatial frequency, say, $20 \mathrm{c} / \mathrm{deg}$, having a period length of only 3 min arc, this means that spatial frequency discrimination can be successfully performed, although the period lengths of two gratings differ by only $3.6-7.2 \mathrm{sec}$ arc. Spatial frequency discrimination can thus be thought of as a form of hyperacuity (Westheimer, 1975), because the smallest resolvable difference is approximately a factor of 10 less than that of the average spacing between the centers of the foveal cones (Hirsch \& Hylton, 1984; Williams \& Collier, 1983). Thomas (1983) has shown that, near contrast threshold, the detection of a grating and the identification of its spatial frequency are related by a proportionality rule: for a given nearthreshold contrast level and spatial-frequency difference the probability that the spatial frequency of the grating will be correctly identified

*To whom all correspondence should be addressed. is associated with a certain probability that the grating will be detected. This finding suggests that the detection of a grating and the identification of its spatial frequency are performed by the same mechanisms.

It is well known that the visual system is less sensitive to rapidly moving grating stimuli. The temporal modulation transfer function of the human visual system has a maximum between 5-10 Hz and falls off rapidly for temporal frequencies above $10 \mathrm{~Hz}$ (Kelly, 1961; Robson, 1966; Watson, 1986). The present study investigates whether the detection and spatialfrequency discrimination of gratings would, in a parallel fashion, covary with drift frequency. If the same mechanisms are involved in the detection and discrimination of spatial frequency then stimulus motion should affect both types of performance in a similar manner. We here report the findings of experiments in which the spatial-frequency discrimination of suprathreshold drifting gratings was measured. The gratings had a wide range of drift rates and three different spatial frequencies. We also explore the effect of grating orientation on the spatial-frequency discrimination of drifting gratings. The results indicate that spatialfrequency discrimination thresholds for gratings 
of medium-high spatial frequency increase for drift rates above $14 \mathrm{~Hz}$. On the contrary, the spatial-frequency discrimination of a low spatial frequency grating $(1 \mathrm{c} / \mathrm{deg})$ is unaffected by drift frequencies up to $40 \mathrm{~Hz}$.

\section{METHOD}

The sinewave luminance gratings used in the experiments were presented on a high-resolution cathode ray tube (Joyce Electronics, Cambridge, U.K.) with white (P4) phosphor and a frame rate of $100 \mathrm{~Hz}$. The control voltages determining the spatial frequency, contrast, spatial position and temporal frequency of the gratings were under the control of a microprocessor. The display had a space-average luminance of $60 \mathrm{~cd} / \mathrm{m}^{2}$. It was masked by a back-illuminated semi-circular surround to a rectangular field of $10 \times 15 \mathrm{deg}$ at $114 \mathrm{~cm}$ viewing distance. The mean luminance and color temperature of the surround were adjusted to closely match that of the display. Precautions were taken to minimize the effects of stray light from the surround onto the screen. Photometric measurements were performed on a regular basis to control the linearity of the voltagecontrast characteristic of the display for the contrast range used.

Spatial-frequency discrimination thresholds were determined using a two-interval forcedchoice procedure. Sinewave gratings were presented in two temporal intervals. The spatial frequency of the one of the gratings was incremented by a defined amount $(\Delta f)$. The subject's task was to discriminate which grating had a higher spatial frequency. The contrast of the gratings was incremented and decremented as a Gaussian function of time. The standard deviation of the temporal Gaussian envelope was $50 \mathrm{msec}$ and total presentation time was $300 \mathrm{msec}$. The two stimuli were separated in time by $1.0 \mathrm{sec}$. These two intervals were announced by computer-generated tones $100 \mathrm{msec}$ prior to the beginning of each stimulus presentation. The test gratings drifted at rates varying between 1.1 and $40 \mathrm{~Hz}$. The direction of drift (leftward vs rightward) was randomized by the computer over presentations.

Contrast thresholds were also measured at each drift frequency for the two observers tested. Contrast detection and spatial-frequency discrimination thresholds were determined using a maximum-likelihood search algorithm (the Best-PEST, Lieberman \& Pentland, 1982). Each measurement was initiated with a parametric value (i.e. contrast or delta spatial frequency) which was roughly 3-4 times that of the expected threshold value. By pressing one of two switches the observer communicated his reponse to the computer and the parametric value was sought that would produce a $75 \%$ correct response rate for the task under investigation. The experimental parameter was controlled using a 20-step single staircase with a resolution of $1 \mathrm{~dB}$ in contrast or $1 \%$ spatial-frequency difference for the contrast detection and frequency discrimination tasks, respectively. A total of 40 trials were conducted for each threshold measurement. Approximately 30 trials suffice for reliable threshold estimates using this procedure (Lieberman \& Pentland, 1982). The results shown in the figures are based on the average of two or more such threshold measurements. The standard error of the parametric estimation in most cases did not exceed $2 \mathrm{~dB}$ for contrast thresholds or $2 \%$ for spatialfrequency discrimination thresholds. Three spatial frequencies $(1,4$ and $12 \mathrm{c} / \mathrm{deg}$ ) and $10 \mathrm{drift}$ frequencies $(1.1-40 \mathrm{~Hz})$ were used. For the spatial-frequency discrimination task, the contrast of the gratings was adjusted to be $1 \mathrm{log}$ unit above its respective detection threshold. The contrast of the gratings varied from 2 to $95 \%$. Owing to its high contrast threshold, for the highest drift frequency used $(40 \mathrm{~Hz})$ grating contrast was less than $1 \log$ unit above the respective contrast threshold for each spatial frequency tested. However, both subjects reported that these gratings were clearly visible and that the apparent contrast of the drifting gratings was similar to that of the gratings drifting at lower rates.

To reduce the effects of fixed spatial and temporal cues over time, we designed the program controlling the experiment in such a way as to add or subtract for each stimulus presentation a random value ("jitter") to such stimulus parameters as grating contrast, temporal frequency, the initial spatial phase of the test gratings, as well as the reference spatial frequency itself. This manipulation appeared to be important since the subject could have otherwise based his judgements on such spurious cues as the apparent contrast or velocity of the reference and test gratings, which would vary systematically with spatial frequency. The maximum size of such randomized jitter never exceeded $10 \%$ of the base value and these values 
averaged to approx. 0 over 40 trials, so as not to bias the average value over time.

In a subsidiary set of experiments we varied the orientation of the test gratings from vertical to horizontal in $15 \mathrm{deg}$ steps. The base spatial frequency in this experiment was $1 \mathrm{c} / \mathrm{deg}$ and the drift frequency was either 5 or $20 \mathrm{~Hz}$. In a further experiment we measured spatial-frequency discrimination thresholds for gratings oriented at $45 \mathrm{deg}$ and drifting at rates varying from 1.1 to $40 \mathrm{~Hz}$. The spatial frequency in this experiment was either 4 or $12 \mathrm{c} / \mathrm{deg}$.
The observers were two of the authors (MWG and JG). JG is an emmetrope and MWG is optimally refracted for his myopia and astigmatism. Both subjects have had extensive experience with similar psychophysical experiments. Therefore, no noticeable effect of learning was evident over the period of experimentation.

\section{RESULTS}

\section{Contrast sensitivity to drifting gratings}

Figure 1 presents the findings of the experiments where we measured the contrast sensi-
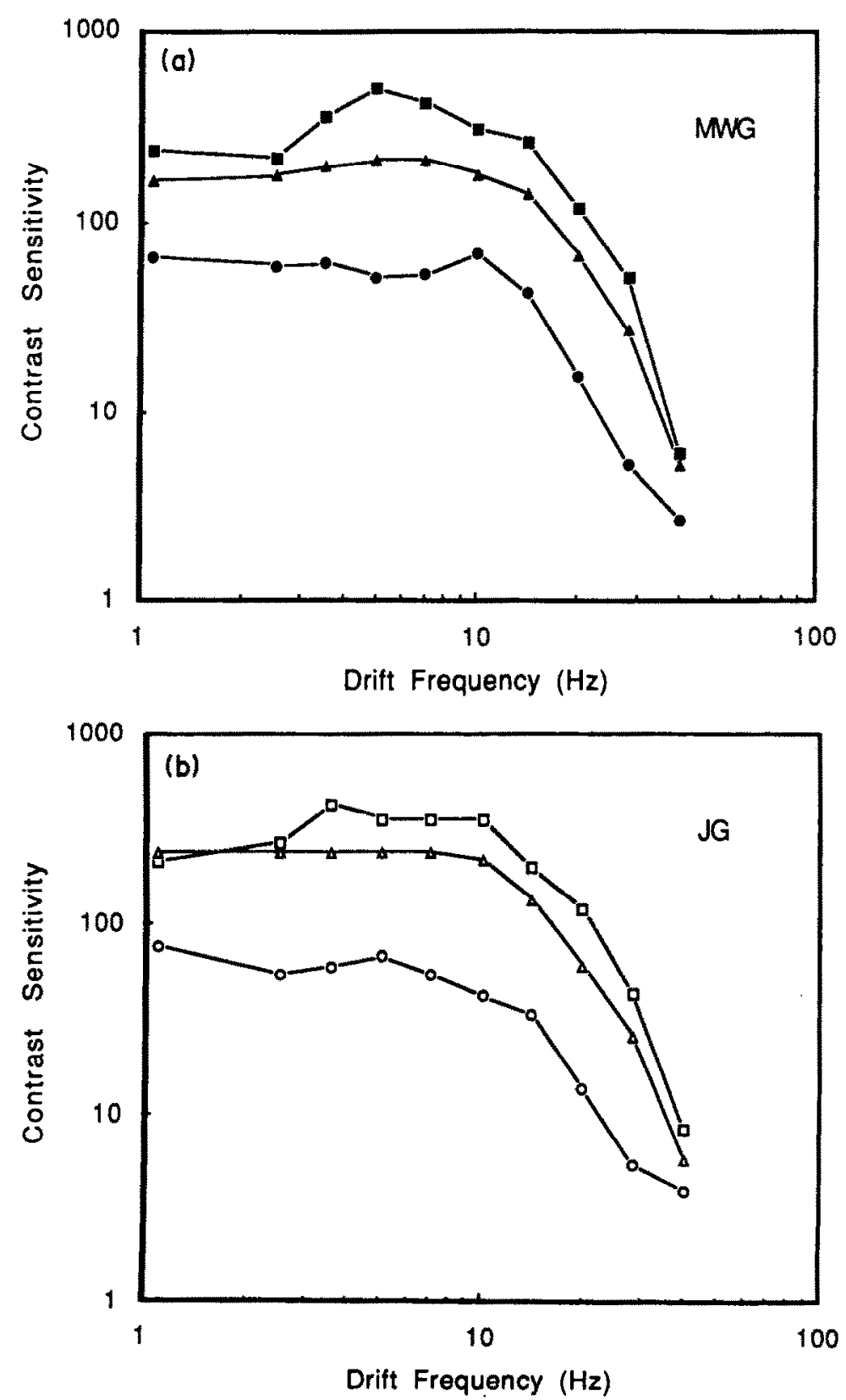

Fig. 1. Contrast sensitivity ( $1 /$ threshold contrast) is plotted as a function of the drift frequency of briefly presented ( $300 \mathrm{msec}$ ) sinewave gratings. Results for threc spatial frequencies (squares $1 \mathrm{c} / \mathrm{deg}$, triangles $4 \mathrm{c} / \mathrm{deg}$, circles $12 \mathrm{c} / \mathrm{deg}$ ) are shown. (a) Presents the findings for observer MWG and (b) for JG. 
tivity to gratings drifting at different rates. The parameter is the spatial frequency of the gratings (1, 4 and $12 \mathrm{c} / \mathrm{deg})$. The findings for subject MWG are shown in Fig. I(a) and those for JG in Fig. 1(b). For both observers, contrast sensitivity peaks around $5 \mathrm{~Hz}$ and falls off rapidly above $10 \mathrm{~Hz}$. In accordance with earlier reports (Robson, 1966; Watson, 1986) the temporal modulation transfer function shows a low-pass characteristic for medium-to-high spatial frequencies and a bandpass characteristic for low spatial frequencies.

\section{Spatial-frequency discrimination of drifting gratings}

Figure 2 presents the findings of the experiments where we measured the spatial-frequency discrimination for gratings having a reference spatial frequency of $1 \mathrm{c} / \mathrm{deg}$. As mentioned above, the contrast of the gratings was adjusted to be approx. $1 \log$ unit above the respective contrast threshold for each drift frequency tested. The Weber fraction of spatial-frequency discrimination, or the ratio of the difference frequency to the reference frequency $(\Delta f / f)$, is plotted as a function of the drift rate of the gratings. Results for MWG are shown by solid, those of JG by open symbols. Spatial frequency discrimination thresholds are fairly constant over a large range of drift frequencies. Values for MWG vary from 2 to $6 \%$ and those for JG from 5 to $8 \%$. Observer MWG exhibited slightly lower discrimination thresholds for medium temporal frequencies.

We next explored the spatial frequency discrimination of gratings having a reference spatial frequency of $4 \mathrm{c} / \mathrm{deg}$. The results for observer MWG and JG are presented in Fig. 3. For this medium spatial frequency, both subjects exhibit discrimination thresholds which are more or less constant up to around $14 \mathrm{~Hz}$. However, for drift frequencies beyond $14 \mathrm{~Hz}$, the Weber fraction increases substantially. MWG shows a change from $6 \%$ at $14.1 \mathrm{~Hz}$ to $24 \%$ at $40 \mathrm{~Hz}$ and JG indicates a threshold change from $6 \%$ at $20 \mathrm{~Hz}$ to $25 \%$ at $40 \mathrm{~Hz}$ drift frequency. This increase in discrimination threshold occurred despite the fact that grating contrast was adjusted to remain a constant amount above threshold (see Method). The observers reported that, although the grating was clearly visible as a grating, they had difficulty discriminating between the reference and test gratings with respect to their spatial frequency.

In the next experiment we increased the reference spatial frequency to $12 \mathrm{c} / \mathrm{deg}$. To compensate for the resolution limits of the display, in this experiment the viewing distance was increased to $228 \mathrm{~cm}$. The increase in viewing distance decreased the angular subtense of the display at the eye to $5 \times 7.5 \mathrm{deg}$. The same viewing distance was used to measure contrast

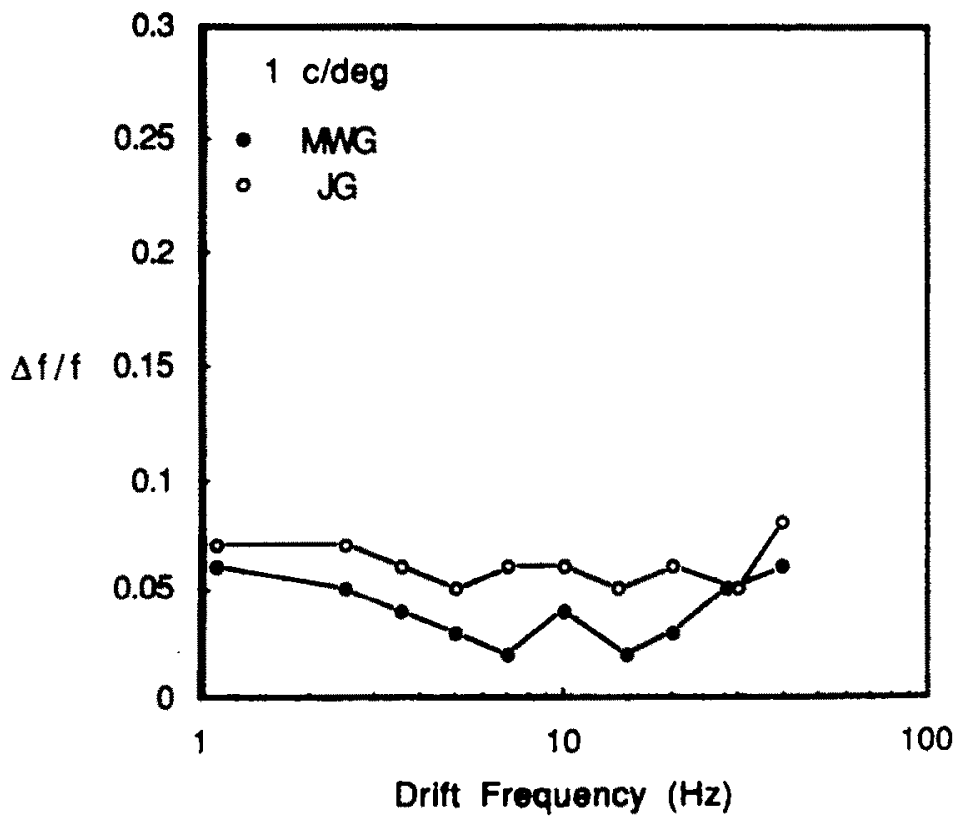

Fig. 2. Spatial-frequency dixcrimination threaholds $(\Delta f / f)$ are presented as a function of the drift frequency of the grating. The spatial frequency of the reference ganting was $1 \mathrm{c} / \mathrm{deg}$. Renults for MWG shown by solid, those for JG by open symbols. 


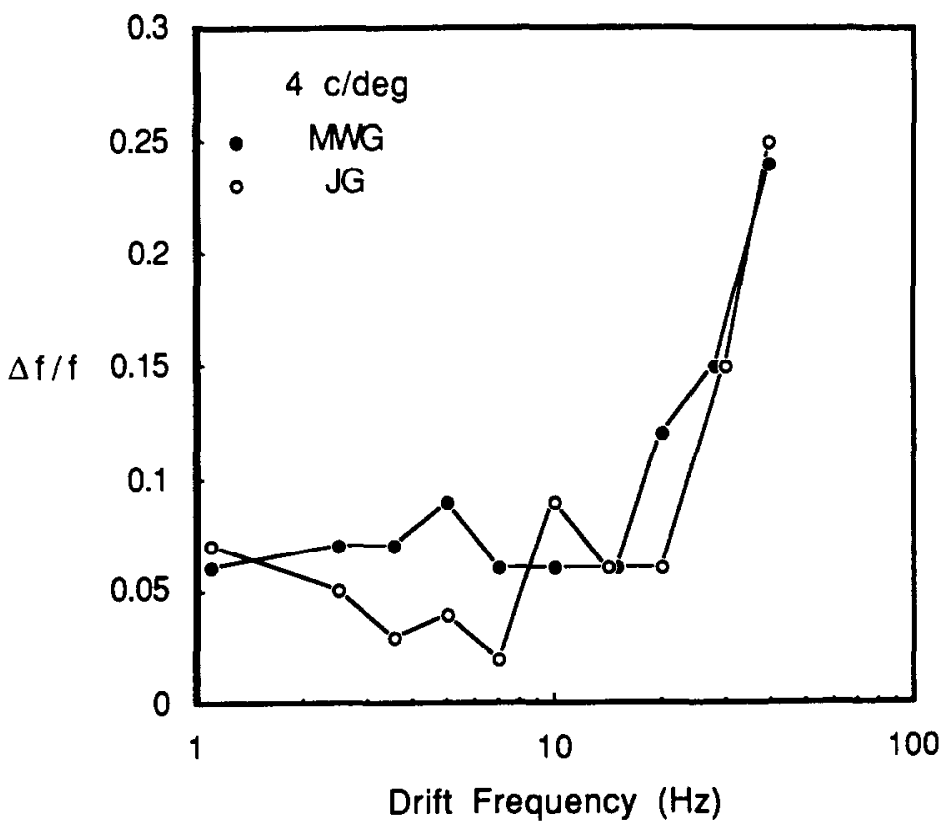

Fig. 3. Spatial-frequency discrimination thresholds as a function of drift frequency for a reference spatial frequency of $4 \mathrm{c} / \mathrm{deg}$.

sensitivity for the $12 \mathrm{c} / \mathrm{deg}$ condition. All other stimulus parameters remained as before. The results are shown in Fig. 4 for the two subjects tested. Here again, we observe good performance for medium-to-low drift rates and poor performance for high drift rates. Note that the transition in thresholds for medium to high drift rates occurs rather abruptly.
Spatial-frequency discrimination of obliquely oriented gratings

Figure 5 presents the results of the experiment in which we explored the effect of grating orientation on the spatial-frequency discrimination of drifting gratings. In Fig. 5, the Weber fraction is plotted as a function of the orientation of

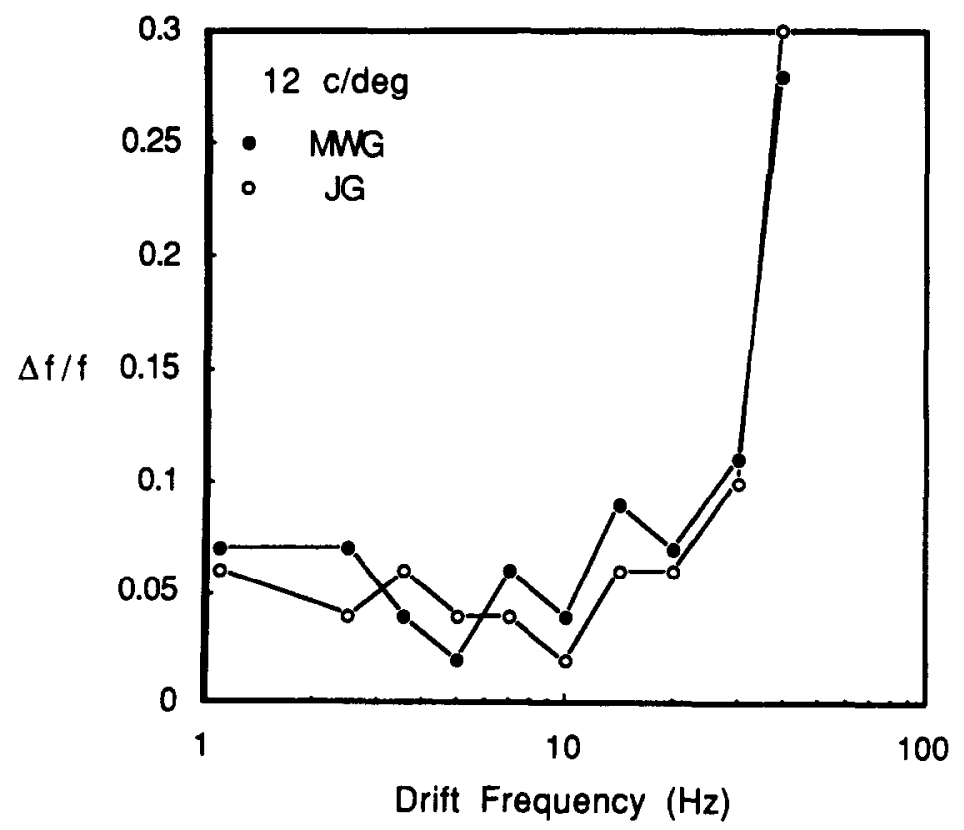

Fig. 4. Spatial-frequency discrimination thresholds as a function of drift frequency for a reference spatial frequency of $12 \mathrm{c} / \mathrm{deg}$. 


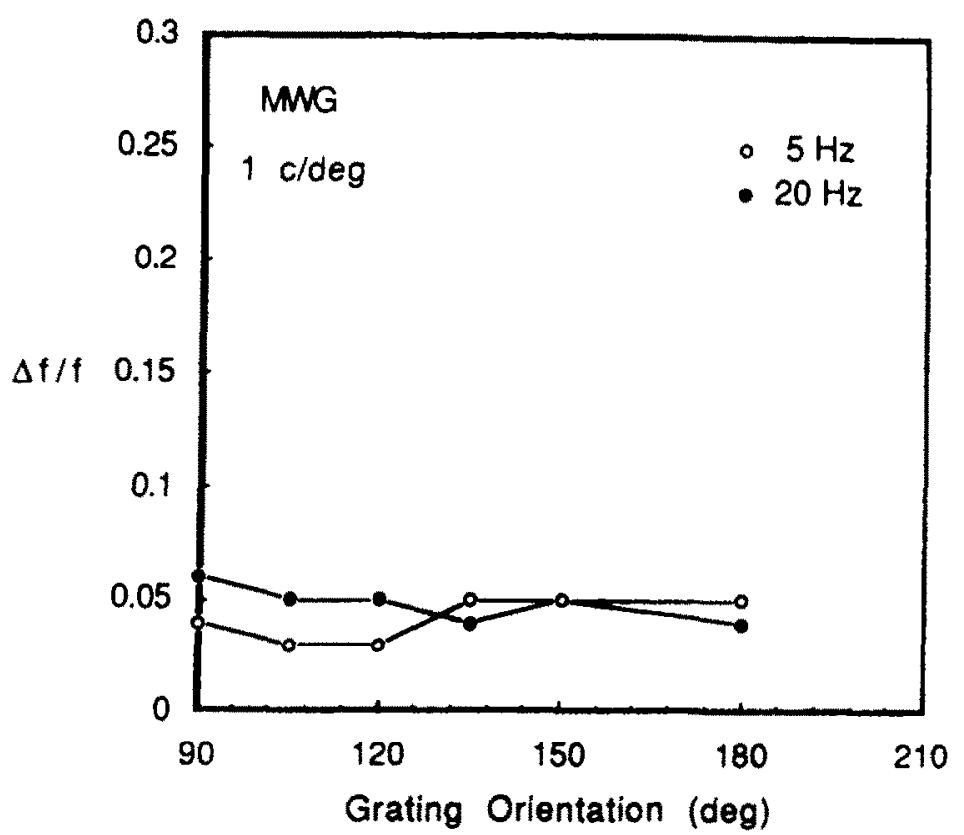

Fig. 5. Spatial-frequency discrimination threaholds as a function of the orientation of the gratings. The spatial frequency of the reference grating was $1 \mathrm{c} / \mathrm{deg}$ and the drif frequency was either $5 \mathrm{~Hz}(\mathrm{O})$ or $20 \mathrm{~Hz}(\mathrm{O})$.

a grating with a $1 \mathrm{c} / \mathrm{deg}$ base frequency and a contrast that was one log unit above detection threshold. The parameter corresponds to the drift frequency of the grating (open circles $5 \mathrm{~Hz}$, solid circles $20 \mathrm{~Hz}$ ). The results indicate that spatial-frequency discrimination performance is independent of the orientation of the drifting grating for this low spatial frequency. They also indicate that there is no interaction between the orientation of the grating and drift frequency.

We further tested whether the increase in discrimination thresholds found for medium-tohigh spatial frequency gratings and high drift rates would be more pronounced for obliquely oriented gratings. Figure 6 compares discrimination thresholds for vertically oriented and obliquely oriented gratings as a function of the drift frequency. For the sake of comparison, the results presented in Figs 3 and 4 for vertical gratings are depicted by the curves in Fig. 6 . The symbols show the results for the condition where the gratings were oriented at $45 \mathrm{deg}$ for a 4 (triangles) and $12 \mathrm{c} / \mathrm{deg}$ (circles) reference spatial frequency. The findings indicate that there is no marked difference between performance for vertical and oblique gratings.

\section{DISCUSSION}

The present findings indicate that spatial-frequency discrimination of low spatial-frequency gratings is unimpaired by stimulus motion for a wide range of drift frequencies (Fig. 2). Contrary to this finding, spatial-frequency discrimination is substantially impaired for gratings having a reference frequency of $4 \mathrm{c} / \mathrm{deg}$ and greater, whenever the drift rate of these gratings exceeds $15 \mathrm{~Hz}$ (Figs 3 and 4). For the two higher spatial frequencies tested (4 and $12 \mathrm{c} / \mathrm{deg}$ ) the temporal frequency, where discrimination performance begins to break down, corresponds to the point in the contrast transfer function where sensitivity begins to decline rapidly (Fig. 1).

It might be argued that eye movements may be causing the effect at spatial frequencies of $4 \mathrm{c} / \mathrm{deg}$ and above. A low spatial frequency grating could be followed easier and thus drift would have less effect on discrimination thresholds. Certain precautions were taken, however, to reduce the effects of pursuit eye movements and spurious cues due to stimulus motion and changes in spatial frequency. First, we used short stimulus durations and random stimulus directions (leftward or rightward drift) to reduce the effects of smooth pursuit eye movements. The grating were only visible for approx. $150 \mathrm{msec}$ and this duration is less than that usually required to voluntarily initiate eye movements (Fischer, 1987). We can thus be fairly certain that eye movements could not have been reliably made by the subjects to reduce image motion on the retina thereby 


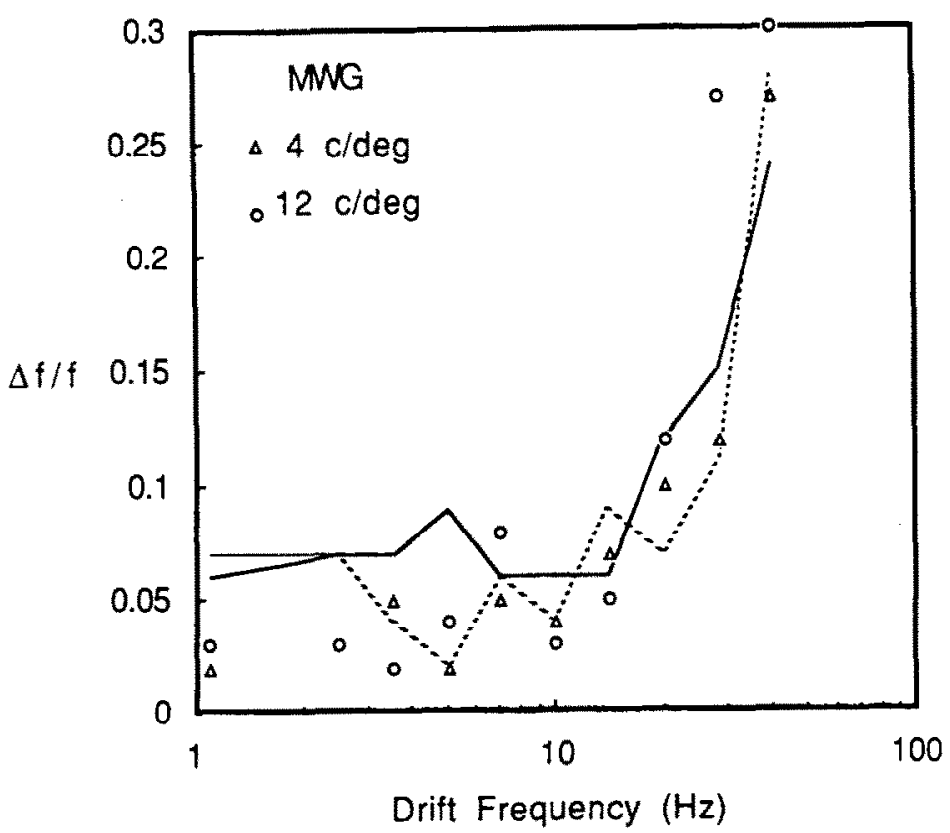

Fig. 6. Spatial-frequency discrimination thresholds as a function of drift frequency for gratings oriented at $45 \mathrm{deg}$. Results for a $4 \mathrm{c} / \mathrm{deg}$ reference shown by triangles, those for a $12 \mathrm{c} / \mathrm{deg}$ reference grating by circles. Continuous $(4 \mathrm{c} / \mathrm{deg}$ ) and broken $(12 \mathrm{c} / \mathrm{deg}$ ) lines show for comparison the results (data points omitted) for gratings of vertical orientation.

improving thresholds for low spatial frequencies. The results that the orientation of the grating had no effect on discrimination threshold further argues against a role of eye movements as a possible contaminating factor in these results.

\section{Effect of grating orientation on spatial-frequency discrimination thresholds}

Westheimer and McKee $(1975,1977)$ have shown that discrimination thresholds for the offset of two vertically oriented lines remains unaffected by image motion for a range of velocities between 0.5 and $3.5 \mathrm{deg} / \mathrm{sec}$. Interestingly, vernier-resolution thresholds were affected by oblique motion, when the vertical targets were moved along the 45 and $135 \mathrm{deg}$ meridians. Compared to thresholds for horizontal motion, vernier-offset thresholds for oblique motion began to diverge for stimulus velocities greater than $2 \mathrm{deg} / \mathrm{sec}$. In contrast, the present results indicate that the good discrimination performance exhibited for low spatial frequency gratings is unaffected by grating orientation (Fig. 5). In addition, the effect of drift frequency on spatial-frequency discrimination for medium-high spatial frequencies was the same for vertically and obliquely oriented gratings (Fig. 6). An important difference between the present experiments and those of Westheimer and McKee (1977) is that the information required to solve the spatial-frequency discrimination task was oriented orthogonal to the direction of motion, whereas the vernier offset cues were oriented obliquely to the axis of stimulus displacement. Such differences appear to be critical for discrimination performance.

\section{Discrimination performance and stimulus motion}

For the vernier targets, Westheimer and McKee (1975) restricted their observations to stimulus velocities of $3.5 \mathrm{deg} / \mathrm{sec}$ and less. In a recent report, Morgan and Benton (1989) replicated the findings of Westheimer and McKee and expanded their observations to stimulus velocities up to $6 \mathrm{deg} / \mathrm{sec}$. Morgan and Benton (1989) did, however, find an effect of stimulus motion for another form of hyperacuityspatial interval discrimination-for two parallel line segments separated, on average, by $4.5 \mathrm{~min}$ arc. By varying the standard interval between the two line segments from 4.5 to $36 \mathrm{~min}$ arc, these authors could explore the interaction between line separation and stimulus velocity. They found that stimulus motion had little effect on width discriminations for widely separated line segments, but did attenuate performance for closely spaced stimuli (Morgan \& Benton, 1989; Fig. 2). The present results indicate that stimulus motion had no effect on spatial-fre- 
quency discrimination thresholds for low spatial frequencies $(1 \mathrm{c} / \mathrm{deg})$, but had a large effect for medium-high spatial frequencies. Thus, the findings of Morgan and Benton (1989) for spatialinterval discrimination of two line segments and our present observations appear to be related to the same underlying mechanisms. Stimulus velocity affects discrimination of line separation or grating spatial frequency when the critical information is contained in the medium-high spatial frequency range and has little effect when this information is stored in the low spatial frequency range. The results of computer simulations in our laboratory suggest that this might be caused by mechanisms having a broader spatial-frequency tuning to high stimulus velocities (or high temporal frequencies). An increase in the spatial-frequency bandwidth of the underlying neural mechanism would lead to a decrease in the differential output of these mechanisms and, as a consequence, to an increase in the spatial-frequency discrimination threshold. This decrease in discrimination efficiency can be thought of as a form of motion blurring (Morgan \& Benton, 1989), but might reflect the simple fact that rapidly moving stimuli are encoded by neural mechanisms with broader spatial-frequency bandwidths.

It is tempting here to speculate on the neural origin of such mechanisms. It has been shown, both empirically (Andrews \& Pollen, 1979; DeValois, Albrecht \& Thorell, 1982; Glezer, Gauzelman \& Yakolev, 1989) and theoretically (Kulikowski, Marcelja \& Bishop, 1982; Marcelja, 1980), that the number of inhibitory and excitatory subunits of simple and complex cells in area $\mathrm{V} 1$ of cat and monkey cortex is related to the sharpness of their spatialfrequency tuning curves (i.e. bandwidth). Mechanisms tuned to medium-high spatial frequencies and low temporal frequencies might have a narrower spatial-frequency bandwidth (and larger number of subunits) than those tuned to medium-high spatial frequencies and high temporal frequencies. The differential responses of single cells in cat cortex in some cases code a difference of $5 \%$ in the spatial frequency of two stationary gratings (Bradley, Skottun, Ohzawa, Sclar \& Freeman, 1987). It would be interesting to see whether this performance is attenuated by high drift rates.

In summary, stimulus motion only interrupts the normally high discrimination performance of the visual system when the drift rate of periodic gratings exceed $15 \mathrm{~Hz}$. Below this value the human visual system is remarkably capable of detecting minute differences in the spatial frequency of two suprathreshold gratings. Above $15 \mathrm{~Hz}$ the fidelity of the visual system begins to break down at least for spatial frequencies of $4 \mathrm{c} / \mathrm{deg}$ and above. We suggest that this change in discrimination efficiency is related to an increase in the spatial-frequency bandwidth of mechanisms sensitive to high temporal frequencies.

Acknowledgements - This research was supported by the Deutsche Forschungsgemeinschaft (SFB 325, B3 and BA). The authors would like to thank Drs $S$. Magnussen and L. Spillmann for critical comments on an earlier version of this manuscript.

\section{REFERENCES}

Andrews, B. W. \& Pollen. D. A. (1979). Relationship between spatial frequency selectivity and receptive field profile of simple cells. Journal of Physiology, London, 287, 163-176.

Bradley, A., Skottun, B. C., Ohzawa, I., Sclar, G. B Freeman, R. D. (1987). Visual orientation and spatial frequency discrimination: A comparison of single neurons and behavior. Journal of Neurophysiology, 57, 755-772.

Campbell, F. W., Nachmias, J. \& Jukes, J. (1970). Spatialfrequency discrimination in human vision. Journal of the Optical Society of America, 60, 555-559.

DeValois, R. L., Albrecht, D. G. \& Thorell, L. G. (1982). Spatial frequency selectivity of cells in macaque visual cortex. Vision Research, 22, 545-559.

Fischer, B. (1987). The preparation of visually guided saccades. Reviews of Physiology. Biochemistry and Pharmacology, 106, 1-35.

Glezer, V. D., Gauzelman, V. E. \& Yakovlev, V. V. (1989). Spatial organization of subfields in receptive felds of cells in cat striate cortex. Vision Research, 29, 777-788.

Hirsch, J. \& Hylton, R. (1982). Limits of spatial-frequency discrimination as evidence of neural interpolation. Jour nal of the Optical Society of America, 72, 1367-1374.

Hirsch, J. \& Hylton, R. (1984). Quality of the primate photoreceptor lattice and limits to spatial vision. Vision Research, 24, 347-355.

Kelly, D. H. (1961). Visual responses to time-dependent simuli. I. Amplitude sensitivity measurements. Journal of the Optical Society of America, 51, 422-429.

Kulikowski, J. J., Marcelja, S. \& Bishop, P. O. (1982). Theory of spatial position and spatial frequency relations in the receptive fields of simple cells in the visual cortex. Biological Cybernetics, 43, 187-198.

Lieberman, H. R. \& Pentland, A. P. (1982). Microcomputer-based estimation of psychophysical thresholds: The best PEST. Behavioral Research Methods and Instrumeniation, 14, 21-25.

Marcelja, S. (1960). Mathematical description of the responses of simple cortical cells. Joumal of the Optical Society of America, $70,1297-1300$.

Mayer, M, J. Kim, C. B. Y. (1986). Smooth frequency discrimination functions for foveal. high-contrast, midspatial frequencies. Joumal of the Optical Sociesy of America A, 3, 1957-1969.

Morgan, M. J. \& Benton, S. (1989). Motion-deblurring in human vision. Nature, London, 340, 385-386. 
Robson, J. G. (1966). Spatial and temporal contrast sensitivity functions of the visual system. Journal of the Optical Society of America, 56, 1141-1142.

Thomas, J. P. (1983). Underlying psychometric function for detecting gratings and identifying spatial frequency. Journal of the Optical Society of America, 73, 751-758. Watson, A. B. (1986). Temporal sensitivity. In Boff, K. R., Kaufman, L. \& Thomas, J. P. (Eds.), Handbook of perception and human performance. Sensory processes and perception (Vol. 1, pp. 6-1-6-43). New York: Wiley.
Westheimer, G. (1975). Visual acuity and hyperacuity. Investigative Ophthalmology, 14, 570-572.

Westheimer, G. \& McKee, S. P. (1975). Visual acuity in the presence of retinal-image motion. Journal of the Optical Society of America, 65, 847-850.

Westheimer, G. \& McKee, S. P. (1977). Integration regions for visual hyperacuity. Vision Research, 17, 89-93.

Williams, D. R. \& Collier, R. (1983). Consequences of spatial sampling by a human photoreceptor mosaic. Science, New York, 221, 499-501. 